\title{
Lymphangioma Circumscriptum
}

National Cancer Institute

\section{Source}

National Cancer Institute. Lymphangioma Circumscriptum. NCI Thesaurus. Code C45485.

A localized lymphang ioma characterized by microcystic changes. 